\title{
PAPER
}

Check for updates

Cite this: Phys. Chem. Chem. Phys. 2017, 19, 15172

Received 9th March 2017, Accepted 10th May 2017

DOI: $10.1039 / c 7 c p 01526 g$

rsc.li/pccp

\section{Diacetylene polymerization on a bulk insulator surface $\dagger$}

\author{
A. Richter, ${ }^{a}$ V. Haapasilta, ${ }^{b}$ C. Venturini, ${ }^{c}$ R. Bechstein, ${ }^{a}$ A. Gourdon, (D) ${ }^{c}$ \\ A. S. Foster*bd and A. Kühnle (iD *a
}

\begin{abstract}
Molecular electronics has great potential to surpass known limitations in conventional silicon-based technologies. The development of molecular electronics devices requires reliable strategies for connecting functional molecules by wire-like structures. To this end, diacetylene polymerization has been discussed as a very promising approach for contacting single molecules with a conductive polymer chain. A major challenge for future device fabrication is transferring this method to bulk insulator surfaces, which are mandatory to decouple the electronic structure of the functional molecules from the support surface. Here, we provide experimental evidence for diacetylene polymerization of 3,3'-(1,3-butadiyne-1,4diyl)bisbenzoic acid precursors on the (10.4) surface of calcite, a bulk insulator with a band gap of around $6 \mathrm{eV}$. When deposited on the surface held at room temperature, ordered islands with a $(1 \times 3)$ superstructure are observed using dynamic atomic force microscopy. A distinct change is revealed upon heating the substrate to $485 \mathrm{~K}$. After heating, molecular stripes with a characteristic inner structure are formed that excellently match the expected diacetylene polymer chains in appearance and repeat distance. The corresponding density functional theory computations reveal molecular-level bonding patterns of both the $(1 \times 3)$ superstructure and the formed striped structure, confirming the assignment of on-surface diacetylene polymerization. Transferring the concept of using diacetylene polymerization for creating conductive connections to bulk insulator surfaces paves the way towards application-relevant systems for future molecular electronic devices.
\end{abstract}

\section{Introduction}

On-surface synthesis holds great promise for creating future molecular electronics devices directly on the supporting surface. ${ }^{1,2}$ While individual molecules acting, e.g., as rectifiers ${ }^{3}$ or switches, ${ }^{4-6}$ have been presented, the connection and contacting of these functional units remain great challenges. Initiating diacetylene polymerization of precursor molecules on surfaces has been recognized as a very promising concept for creating conductive polymer chains to connect functional molecular units. ${ }^{7,8}$ In the bulk, the solid-state polymerization of diacetylene derivatives upon heating or light irradiation has been explained by topochemical 1,4-polymerization. ${ }^{9}$ For this reaction, the crystalline

\footnotetext{
${ }^{a}$ Institute of Physical Chemistry, Johannes Gutenberg University Mainz, 55099 Mainz, Germany. E-mail: kuehnle@uni-mainz.de

${ }^{b}$ COMP, Department of Applied Physics, Aalto University, PO Box 11100, FI-00076 Aalto, Finland. E-mail: adam.foster@aalto.fi

${ }^{c}$ CNRS, CEMES, Nanoscience Group, BP 94347, 29 Rue J. Marvig, 31005 Toulouse, France

${ }^{d}$ Division of Electrical Engineering and Computer Science, Kanazawa University, Kanazawa 920-1192, Japan

$\dagger$ Electronic supplementary information (ESI) available: Details on synthesis procedures and computation. See DOI: 10.1039/c7cp01526g
}

arrangement of the precursor monomers has been recognized as being of pivotal importance, as the acetylene units have to be within a distance of no more than $0.4 \mathrm{~nm}$ and the molecular backbones of two diacetylene monomers must form an angle close to $45^{\circ}$ with respect to the crystal translational vector. ${ }^{10}$

On surfaces, suitable precursors have been shown to arrange in a pattern fulfilling the above topochemical requirements. An elegant example of on-surface polymerization initiated by a voltage pulse with the tip of a scanning tunnelling microscope (STM) has been presented by M. Aono and co-worker. ${ }^{11}$ In this seminal study, initiation and termination of the chain polymerization with remarkable nanoscale precision have been presented using 10,12-nonacosadiynoic acid monomers on a graphite surface. To date, on-surface diacetylene polymerization has been reported for a large range of systems, including polymerization at the solid-liquid interface ${ }^{12}$ as well as in air $^{11,13}$ and under ultra-high vacuum (UHV). ${ }^{14,15}$ The majority of these studies have been carried out using a graphite support. ${ }^{8,12,13,16-19}$ Only a few other substrates have been studied for diacetylene polymerization, including, e.g., $\mathrm{Au}(111),{ }^{14} \mathrm{MoS}_{2}(0001),{ }^{8,15}$ hexagonal boron nitride $^{20}$ and epitaxial graphene on $\mathrm{SiC}(0001) .{ }^{21}$ Most importantly, no bulk insulator surface has been used so far. In the view of molecular electronics applications, bulk insulator surfaces are 
(a) Calcite (10.4)

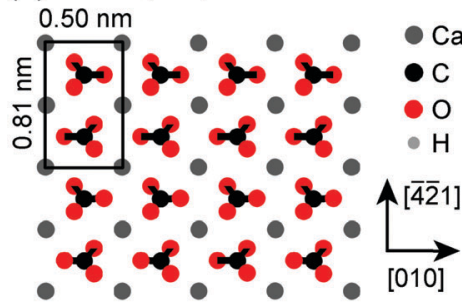

(b) 3BBA

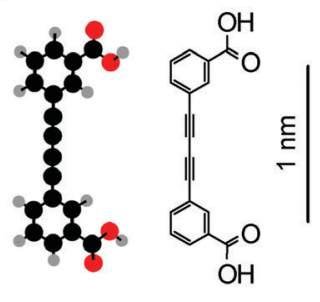

Fig. 1 The calcite (10.4) surface (a) and the 3,3'-(1,3-butadiyne-1,4-diyl)bisbenzoic acid (3BBA) precursor molecule (b) geometries after DFT optimization (with a skeletal formula). The surface unit cell and the perpendicular surface directions are indicated. The molecule is planar. The scale bar applies to both subsets.

highly desired as they offer an easy way for electronic decoupling of the functional molecular structures from the supporting substrate surface. Thus, when aiming for using diacetylene polymerization for creating conductive polymer chains to electrically connect functional units, bulk insulator substrates need to be explored. In this context, calcite has been proven to constitute a very promising substrate ${ }^{22,23}$ with a band gap of $6 \mathrm{eV}^{24}$ In contrast to many other insulator surfaces, the calcite (10.4) surface (Fig. 1a) exhibits a comparatively high surface energy of $590 \mathrm{~mJ} \mathrm{~m}^{-2} .^{22}$ Being ionic, the surface offers the possibility of electrostatically anchoring molecules to the calcium cations or carbonate anions. In addition to this, the protruding oxygen atoms of the carbonate groups offer the possibility of hydrogen bonding. Here, we use 3,3'-(1,3-butadiyne1,4-diyl)bisbenzoic acid (3BBA) molecules (Fig. 1b) as precursors for on-surface diacetylene polymerization.

We use dynamic atomic force microscopy (AFM) operated under UHV to image the molecular islands that self-assemble upon the adsorption of the precursors onto calcite (10.4) held at room temperature. After irradiation with a mercury lamp or after annealing the substrate to $485 \mathrm{~K}$, a new structure is found that exhibits a row-like shape with an internal periodicity that excellently matches the repeat distance expected for a diacetylene polymer chain. The experimental results are accompanied by density functional theory (DFT) computations revealing molecular-level bonding patterns and energetics of the observed structures. This work thus paves the way towards making use of diacetylene polymerization for molecular electronics applications on a bulk insulator surface.

\section{Methods}

\section{Molecule synthesis}

3BBA was prepared from commercial 3-iodobenzoic acid, by analogy with literature procedures ${ }^{25-27}$ After methylation of the carboxylic group, Sonogashira coupling with trimethylsilyl-acetylene gave methyl 3-((trimethylsilyl)ethynyl)benzoate, followed by deprotection by tetra- $n$-butylammonium fluoride to yield methyl 3 -ethynylbenzoate ( $64 \%$ for the two steps). Glaser coupling of the latter gave dimethyl 3,3'-(buta-1,3-diyne-1,4-diyl)dibenzoate in 91\% yield, which was then saponified by lithium hydroxide followed by acidification to yield 3BBA (54\%). See the ESI $\dagger$ for detailed procedures.

\section{Dynamic AFM imaging}

For direct imaging of the molecular structures deposited on the surface of the bulk insulator calcite, we used dynamic AFM operated in the so-called frequency modulation mode under UHV (VT AFM from ScientaOmicron, Taunusstein, Germany). We use standard silicon cantilevers (Nanosensors, Neuchâtel, Switzerland), having a typical eigen frequency of $300 \mathrm{kHz}$ and a force constant of $40 \mathrm{~N} \mathrm{~m}^{-1}$ under UHV. Prior to molecule deposition, a calcite crystal (Korth Kristalle, Altenholz, Germany) was cleaved in situ to prepare a clean (10.4) surface. After cleavage, the crystal is annealed to $640 \mathrm{~K}$ in situ, a routine that has been shown to be necessary for high-resolution imaging. The precursor molecules were deposited using a home-built Knudsen cell. Sublimation of sub-monolayers could be achieved with a cell temperature of around $450 \mathrm{~K}$ and a sublimation time of $25 \mathrm{~min}$.

\section{Computations}

We performed density functional theory (DFT) calculations $(T=0 \mathrm{~K})$ and molecular dynamics (MD) simulations $(T \neq 0 \mathrm{~K})$ using the cp2k program suite. ${ }^{28}$ In all computations, the PBE functional ${ }^{29}$ was used together with the D3 dispersion correction scheme. ${ }^{30}$ The Gaussian Plane Wave Method ${ }^{31}$ was used to solve the electronic structure. Core electronic states were modelled with GTH pseudopotentials ${ }^{32}$ and the valence electrons were described using molecularly optimized polarized double- $\zeta$ basis functions. ${ }^{33}$ The electronic density was described using a plane wave basis with an energy cutoff of 500 Ry. More details can be found in the ESI. $\dagger$

\section{Results and discussion}

\section{Self-assembled structures}

First, we investigated the structural arrangement of sub-monolayer coverage of 3BBA deposited onto a freshly cleaved calcite (10.4). As shown in Fig. 2a, the molecules self-assemble into molecular islands with an elongated shape. The apparent height of the islands is $0.6 \mathrm{~nm}$. The long axis of the islands is oriented along the [010] calcite direction. A zoom into an island (Fig. 2b) shows an inner structure with a $(1 \times 3)$ superstructure forming bright rows along the [010] calcite direction. The analysis reveals that the island patterns consist of either an even or odd number of rows. Furthermore, neighbouring rows are of different lengths and defects can be observed within individual rows in Fig. 2a. Together with the size of the precursor molecule, these AFM data suggest that there are two molecules within the $(1 \times 3)$ superstructure unit cell, that is, individual molecules cause the observed bright features.

To obtain a more detailed molecular-level view of the formed structures, we performed DFT calculations and first-principles molecular dynamics (FPMD) simulations. First, we investigated the adsorption of a single 3BBA molecule onto the calcite (10.4) surface. The computations suggest that the preferred adsorption orientation is flat along the surface with both carboxyl $(\mathrm{COOH})$ groups of the molecule forming hydrogen bonds with the calcite carbonate group oxygen atoms (Fig. 3a). According to the calculations, the adsorption energy is $2.4 \mathrm{eV}$, in line with 

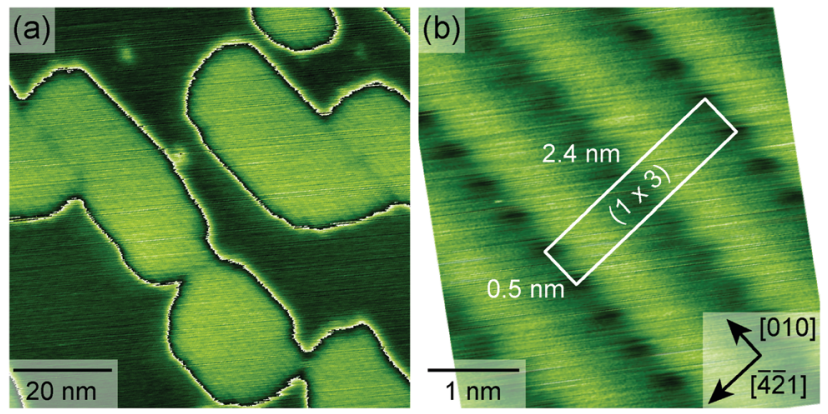

Fig. 2 Self-assembled molecular structures after sub-monolayer sublimation on a freshly cleaved calcite (10.4) surface held at room temperature under UHV. (a) Islands with a striped inner structure appear to self-assemble on the surface. The islands exhibit an elongated shape. (b) A zoom onto an island reveals a $(1 \times 3)$ inner structure. The image is corrected for drift.

room temperature adsorption and earlier calculations with similar molecules on calcite. ${ }^{34}$ In a recent investigation, the biphenyl-4,4'dicarboxylic acid molecule, which also anchors to the surface via two $\mathrm{COOH}$ groups, was calculated to have several adsorption positions on calcite, all with similar energies. ${ }^{34}$ Most probably, 3BBA will also have several stable adsorption geometries with adsorption energies of the order of $2.4 \mathrm{eV}$. The lateral orientation shown in Fig. 3a is the one we consider most relevant towards the observed self-assembly pattern.

However, the flat adsorption geometry adapted by the isolated 3BBA molecule cannot be the orientation of the molecules in the self-assembled pattern: the flat orientation does not fit

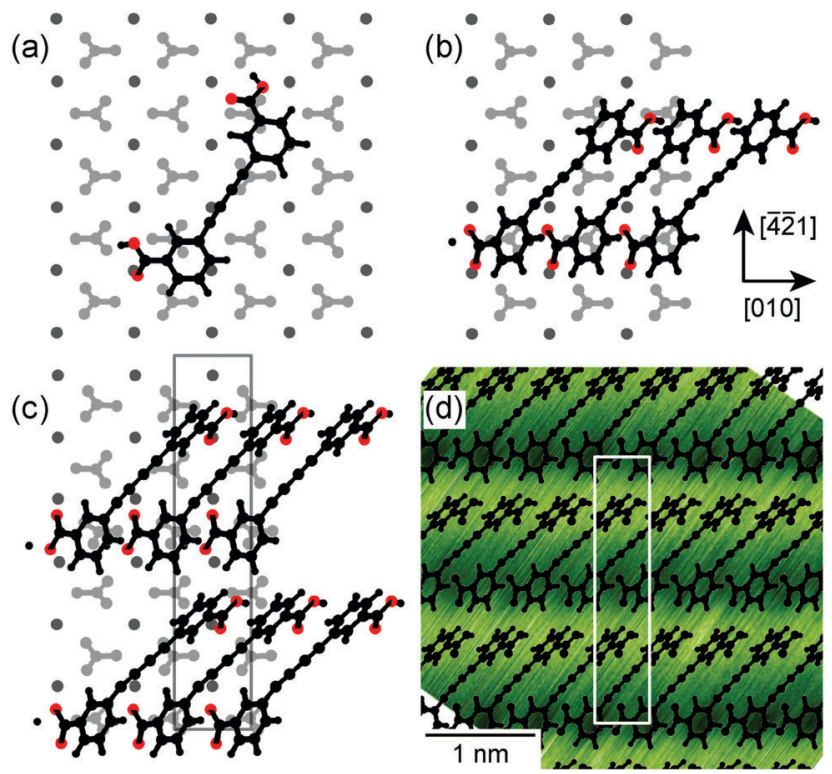

Fig. 3 Optimized isolated 3BBA molecule (a), molecular row of 3BBA molecules (b) and the molecular structure in the observed $(1 \times 3)$ arrangement $(c)$ on the calcite (10.4) surface. In (c) and (d) the $(1 \times 3)$ supercell is indicated. For simplicity, carbonate groups are shown in light and calcium ions in dark grey, carbon atoms of the molecule are larger and hydrogen atoms smaller black spheres while oxygen atoms of the molecule are shown in red. In $(d)$ the $(1 \times 3)$ arrangement is overlaid on top of an AFM image of the molecular island to illustrate the excellent match in size and pattern. Note that the absolute position of the overlay with respect to the image is unknown. into the observed $0.5 \mathrm{~nm}$ repeat distance along the calcite [010] axis. This suggests that the individual molecules must be tilted upwards (see the ESI $\dagger$ ). In the computations, tilted molecular orientation emerges naturally when the molecules are brought close to each other. Fig. 3b shows such an optimized molecular geometry with the observed $(1 \times 3)$ superstructure periodicity, but with half the density, that is, one molecule in the $(1 \times 3)$ superstructure unit cell. Here, the adsorption (or the island formation) energy per molecule is $2.6 \mathrm{eV}$ - there is a small favourable interaction between the molecules. In the optimized geometry, each 3BBA molecule is still forming two hydrogen bonds with the surface. Also, the planes of the two phenyl groups within the molecules form an angle of almost $90^{\circ}$ between each other. Finally, in Fig. 3c an optimised molecular structure corresponding to two molecules in the $(1 \times 3)$ superstructure unit cell is shown. In this case the adsorption/island formation energy per molecule is $2.7 \mathrm{eV}$. Here one of the two molecules in the $(1 \times 3)$ cell has the same bonding pattern as shown in Fig. $3 \mathrm{~b}$ and the additional molecule is placed at 1.5 unit cell lengths along the $[-4-21]$ calcite direction in relation to the previous. Also the additional molecule is bonding via two hydrogen bonds and retains the rotated carbon ring geometry. The calculated $(1 \times 3)$ superstructure is in excellent agreement with the experiments as is shown in the overlay in Fig. 3d (see the ESI $\dagger$ for more details about the considered structures).

\section{Changes induced by annealing}

In order to evaluate whether annealing can induce a structural change, we have stepwise increased the annealing temperature and inspected the surface after annealing. When annealing the molecule-covered substrate at $485 \mathrm{~K}$ for $1 \mathrm{~h}$ three structural changes were observed as can be seen in Fig. 4a.

The number of islands has decreased and small, roundish clusters have formed that are scattered over the surface (see Fig. 4a). Most importantly, single stripes oriented along the [010] direction have also formed. These stripes can be up to $60 \mathrm{~nm}$ long. Most of them appear to be connected to an island, but also separated stripes can be found. When annealing instead at $520 \mathrm{~K}$ for $1 \mathrm{~h}$ the described structural changes advance further. Then, fewer islands are found while more clusters and even longer stripes are formed. After annealing at $555 \mathrm{~K}$ for $1 \mathrm{~h}$, the islands disappear completely and only clusters and stripes are present on the surface (see ESI $\dagger$ ). The fact that the stripes are more stable upon annealing than the islands indicates a stronger molecule-molecule interaction within the stripes as compared to the island structure. These results suggest a covalent linkage of the precursors leading to a one-dimensional structure of superior thermal stability as compared to the self-assembled islands.

From the high-resolution image of a single stripe (Fig. 4b), a periodic structure in the [010] direction with a repeat distance of $0.5 \mathrm{~nm}$, in accordance with the calcite surface, can be determined. This is a strong indication for a successful diacetylene polymerization of the precursors on the (10.4) surface of calcite. To follow this hypothesis, we performed DFT computations starting with the calculated island geometries. We identified several polymerized 

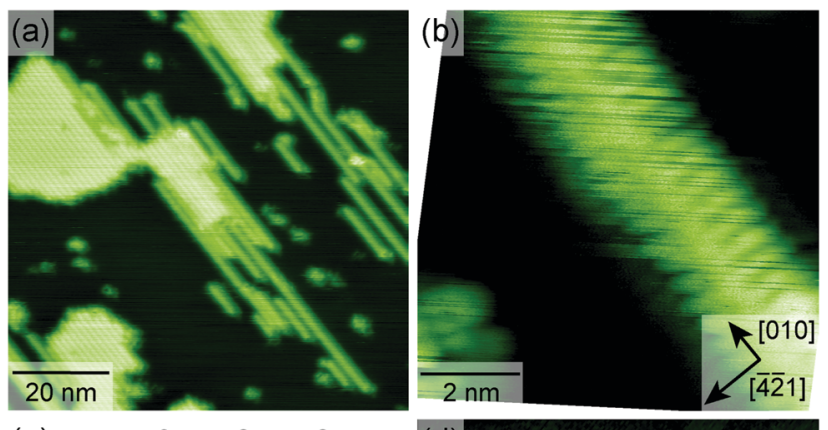

(c)
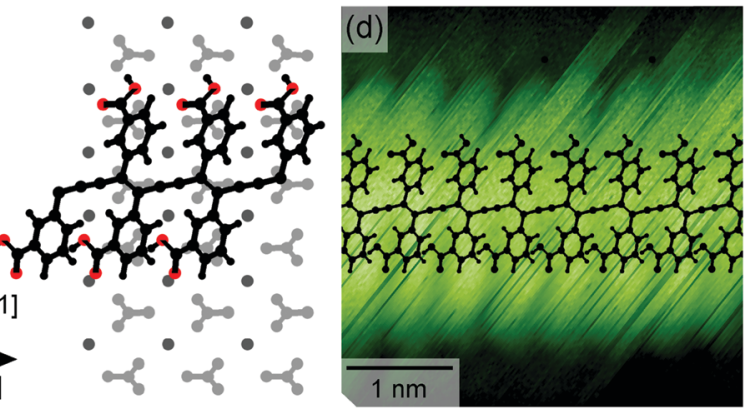

Fig. 4 Molecular structures after annealing. (a) Upon annealing at $485 \mathrm{~K}$ for $1 \mathrm{~h}$, three distinct changes are observed: the number of islands has decreased. Clusters are formed. Most importantly, molecular rows exist on the surface. (b) Zoom onto a chain-like molecular row. (c) Optimized polymerized molecular structure from DFT (colours as in Fig. 3). (d) Zoom onto a chain-like molecular row with a superimposed diacetylene polymer structure from DFT.

covalently bonded structures with a $0.5 \mathrm{~nm}$ repeat distance along the [010] calcite direction. The most stable polymerized structure is shown in Fig. 4c (see the ESI $\dagger$ for more details). According to the optimizations, the polymerized configuration is $1.2 \mathrm{eV}$ more stable (per molecule) than the non-covalently bound $(1 \times 3)$ superstructure.

In the experiments, the apparent height of the stripes is approximately $0.5 \mathrm{~nm}$, i.e., $0.1 \mathrm{~nm}$ lower than the islands. DFT optimization calculations report heights of $0.75 \mathrm{~nm}$ and $0.61 \mathrm{~nm}$ for the islands and polymer stripes, respectively, in qualitative agreement with the experiments. The striped structure of the polymer from DFT calculations is shown as an overlay in Fig. 4d. Again, the agreement between the experiments and theory is good.

The favourable molecule-molecule distance for diacetylene polymerization has been identified to be in the range of $0.47-$ $0.52 \mathrm{~nm}$ with an angle of $45^{\circ}$ for the molecular axis with respect to the crystal translation, ${ }^{10}$ which is perfectly matched in the molecular islands formed prior to annealing: the moleculemolecule distance along the [010] direction is $0.5 \mathrm{~nm}$ as indicated in Fig. 2b. Based on DFT calculations the resulting distance between the diacetylene moieties is $0.36 \mathrm{~nm}$. Therefore, it appears that the self-assembly of 3BBA molecules on the calcite (10.4) surface produces ideal conditions for polymer chain formation along the [010] surface direction.

The reported islands, as well as the stripes, are stable on the surface at room temperature for several days when stored in the UHV chamber. This is further evidence that the observed structural changes were, indeed, induced by the annealing procedure.

The observed clusters can be interpreted as by-products of the on-surface linking reaction. The molecules are obviously able to diffuse on the surface during the heating process and can, therefore, form a variety of reaction products. Moreover, the precursor arrangement favourable to initiate this topochemical reaction is obviously lost as soon as molecules start to diffuse. Consequently, a higher initial coverage - which reduces the molecules's mobility should lead to suppression of by-product formation and an increased yield for stripe formation.

\section{Changes induced by irradiation}

The above-described changes could also be induced by using irradiation with light from a mercury lamp instead of annealing. The full lamp spectrum was tested and also only a part of the spectrum, which was obtained by using a (302 \pm 23$) \mathrm{nm}$ filter. Qualitatively the same results were obtained in both cases (see the ESI $\dagger$ ).

\section{Conclusions}

In conclusion, we present evidence for diacetylene polymerization carried out on a bulk insulator surface. Using dynamic AFM operated under UHV, we show that 3,3'-(1,3-butadiyne-1,4-diyl)bisbenzoic acid precursor molecules deposited on calcite (10.4) form ordered islands with a $(1 \times 3)$ inner structure. Irradiation of the surface with a mercury lamp or annealing the substrate to $485 \mathrm{~K}$ results in a distinct change in the molecular structures. Besides the islands and newly formed clusters, now row-like structures exist on the surface that excellently match the expected polymer chains in appearance and repeat distance. Detailed DFT computations show that the molecular arrangement in the self-assembled islands is suitable for polymerization with an energy gain of $1.2 \mathrm{eV}$ per diacetylene unit formed. It is expected that reducing the molecular mobility by increasing the coverage is beneficial for diacetylene polymerization, as this forces the molecules to stay in the island structure at elevated temperatures, which appears to be favourable for the topochemical reaction.

This work provides experimental and theoretical evidence that the concept of diacetylene polymerization can be transferred to bulk insulator surfaces. This is pivotal for creating conductive polymer chains to connect functional molecular devices for future molecular electronics.

\section{Acknowledgements}

We gratefully acknowledge financial support from the EU through grant PAMS (seventh framework program GA 610446), the Academy of Finland through its Centres of Excellence Program project no. 915804, and the CSC-IT Center for Science Ltd, Espoo, for computational resources.

\section{Notes and references}

1 A. Gourdon, Angew. Chem., Int. Ed., 2008, 47, 6950-6953. 
2 R. Lindner and A. Kühnle, ChemPhysChem, 2015, 16, 1582-1592.

3 A. Aviram and M. A. Ratner, Chem. Phys. Lett., 1974, 29, 277-283.

4 P. Liljeroth, J. Repp and G. Meyer, Science, 2007, 317, 1203-1206.

5 C. Loppacher, M. Guggisberg, O. Pfeiffer, E. Meyer, M. Bammerlin, R. Lüthi, R. Schlittler, J. K. Gimzewski, H. Tang and C. Joachim, Phys. Rev. Lett., 2003, 90, 066107.

6 Z. J. Donhauser, B. A. Mantooth, K. F. Kelly, L. A. Bumm, J. D. Monnell, J. J. Stapleton, D. W. Price, A. M. Rawlett, D. L. Allara, J. M. Tour and P. S. Weiss, Science, 2001, 292, 2303-2307.

7 Y. Okawa, M. Akai-Kasaya, Y. Kuwahara, S. K. Mandal and M. Aono, Nanoscale, 2012, 4, 3013-3028.

8 M. Nakaya, Y. Okawa, C. Joachim, M. Aono and T. Nakayama, ACS Nano, 2014, 8, 12259-12264.

9 G. Wegner, Z. Naturforsch., B: Anorg. Chem., Org. Chem., Biochem., Biophys., Biol., 1969, 24, 824-832.

10 K. Fahsi, J. Deschamps, K. Chougrani, L. Viau, B. Boury, A. Vioux, A. van der Lee and S. G. Dutremez, CrystEngComm, 2013, 15, 4261-4279.

11 Y. Okawa and M. Aono, Nature, 2001, 409, 683-684.

12 P. C. M. Grim, S. De Feyter, A. Gesquière, P. Vanoppen, M. Rücker, S. Valiyaveettil, G. Moessner, K. Müllen and F. C. De Schryver, Angew. Chem., Int. Ed. Engl., 1997, 36, 2601-2603.

13 Y. Okawa, S. K. Mandal, C. Hu, Y. Tateyama, S. Goedecker, S. Tsukamoto, T. Hasegawa, J. K. Gimzewski and M. Aono, J. Am. Chem. Soc., 2011, 133, 8227-8233.

14 O. Endo, T. Furuta, H. Ozaki, M. Sonoyama and Y. Mazaki, J. Phys. Chem. B, 2006, 110, 13100-13106.

15 O. Endo, T. Sera, M. Suhara, H. Ozaki and Y. Mazaki, J. Phys.: Conf. Ser., 2008, 100, 052058.

16 D. Takajo, A. Inaba and K. Sudoh, Langmuir, 2014, 30, 2738-2744.

17 Y. Okawa, D. Takajo, S. Tsukamoto, T. Hasegawa and M. Aono, Soft Matter, 2008, 4, 1041-1047.

18 A. Miura, S. De Feyter, M. M. S. Abdel-Mottaleb, A. Gesquiere, P. C. M. Grim, G. Moessner, M. Sieffert,
M. Klapper, K. Muellen and F. C. De Schryver, Langmuir, 2003, 19, 6474-6482.

19 O. Endo, H. Ootsubo, N. Toda, M. Suhara, H. Ozaki and Y. Mazaki, J. Am. Chem. Soc., 2004, 126, 9894-9895.

20 V. M. Marina, O. Yuji, V. Elisseos, W. Kenji, T. Takashi, J. Christian and A. Masakazu, Nanotechnology, 2016, 27, 395303.

21 A. Deshpande, C.-H. Sham, J. M. P. Alaboson, J. M. Mullin, G. C. Schatz and M. C. Hersam, J. Am. Chem. Soc., 2012, 134, 16759-16764.

22 P. Rahe, M. Kittelmann, J. L. Neff, M. Nimmrich, M. Reichling, P. Maass and A. Kühnle, Adv. Mater., 2013, 25, 3948-3956.

23 P. Rahe, M. Nimmrich and A. Kühnle, Small, 2012, 8, 2968. 24 D. R. Baer and D. L. Blanchard, Appl. Surf. Sci., 1993, 72, 295-300.

25 E. Merkul, D. Urselmann and T. J. J. Müller, Eur. J. Org. Chem., 2011, 238-242.

26 M. H. Vilhelmsen, J. Jensen, C. G. Tortzen and M. B. Nielsen, Eur. J. Org. Chem., 2013, 701-711.

27 H. Matsubara, T. Shimura, A. Hasegawa, M. Semba, K. Asano and K. Yamamoto, Chem. Lett., 1998, 1099-1100.

28 J. VandeVondele, M. Krack, F. Mohamed, M. Parrinello, T. Chassaing and J. Hutter, Comput. Phys. Commun., 2005, 167, 103-128.

29 J. P. Perdew, K. Burke and M. Ernzerhof, Phys. Rev. Lett., 1996, 77, 3865-3868.

30 S. Grimme, J. Antony, S. Ehrlich and H. Krieg, J. Chem. Phys., 2010, 132, 15410.

31 G. Lippert, J. Hutter and M. Parrinello, Mol. Phys., 1997, 92, 477-487.

32 S. Goedecker, M. Teter and J. Hutter, Phys. Rev. B: Condens. Matter Mater. Phys., 1996, 54, 1703-1710.

33 J. VandeVondele and J. Hutter, J. Chem. Phys., 2007, 127, 114105.

34 L. Schüller, V. Haapasilta, S. Kuhn, H. Pinto, R. Bechstein, A. S. Foster and A. Kühnle, J. Phys. Chem. C, 2016, 120, 14730-14735. 